\title{
Mass Spectrometric Separation and Quantitation of Overlapping Isotopologues. $\mathrm{H}_{2} \mathrm{O} / \mathrm{HOD} / \mathrm{D}_{2} \mathrm{O}$ and $\mathrm{H}_{2} \mathrm{Se} / \mathrm{HDSe} / \mathrm{D}_{2}$ Se Mixtures
}

\author{
Juris Meija and Zoltan Mester \\ Institute for National Measurement Standards, National Research Council Canada, Ottawa, Ontario, Canada
}

\author{
Alessandro D’Ulivo \\ Laboratory of Instrumental Analytical Chemistry, Institute for Chemical and Physical Processes, Research \\ area of Pisa, National Research Council of Italy, Pisa, Italy
}

Three conceptually different mathematical methods are presented for accurate mass spectrometric determination of $\mathrm{H}_{2} \mathrm{O} / \mathrm{HOD} / \mathrm{D}_{2} \mathrm{O}$ and $\mathrm{H}_{2} \mathrm{Se} / \mathrm{HDSe} / \mathrm{D}_{2} \mathrm{Se}$ concentrations from mixtures. These are alternating least-squares, weighted two-band target entropy minimization, and a statistical mass balance model. The otherwise nonmeasurable mass spectra of partially deuterated isotopologues (HOD and HDSe) are mathematically constructed. Any recorded isotopologue mixture mass spectra are then deconvoluted by least-squares into their components. This approach is used to study the $\mathrm{H}_{2} \mathrm{O} / \mathrm{D}_{2} \mathrm{O}$ exchange reaction, and is externally validated gravimetrically. The $\mathrm{H}_{2} \mathrm{O} / \mathrm{D}_{2} \mathrm{O}$ exchange equilibrium constant is also measured from the deconvoluted $70 \mathrm{eV}$ electron impact GC/MS data $(K=3.85 \pm 0.03)$. (J Am Soc Mass Spectrom 2006, 17, 1028-1036) (c) 2006 American Society for Mass Spectrometry

$\mathrm{I}$ sotopologues are compounds that differ in isotopic composition only, such as $\mathrm{H}_{2} \mathrm{O}$ and $\mathrm{D}_{2} \mathrm{O}$. These compounds play an important role in analytical chemistry, especially in quantitative analysis where most of the modern internal quantitation methods are based on isotopologues. The mass-domain separation of isotopologues, such as $\mathrm{HBr}$ and $\mathrm{DBr}$, is a trivial task since there is no mass spectral overlap on the ${ }^{81} \mathrm{Br}$ molecular ions of the two species. However, the presence of two hydrogen atoms (as in $\mathrm{H}_{2} \mathrm{O}$ or $\mathrm{H}_{2} \mathrm{Se}$ ) makes the direct estimate of each isotopologue impossible from its electron impact mass spectra. The mass spectrum of $\mathrm{H}_{2} \mathrm{O}$ has two abundant signals at $m / z=18$ and 17, the mass spectrum of $\mathrm{D}_{2} \mathrm{O}$ has two abundant signals at $m / z=20$ and 18. HOD, whose mass spectrum cannot be directly measured, is expected to have three abundant ions at $m / z=19,18$, and 17 . Due to the above-mentioned spectral overlaps, a 1:1 mixture of $\mathrm{H}_{2} \mathrm{O}$ and $\mathrm{D}_{2} \mathrm{O}$ gives a ratio of the molecular ions (at $\mathrm{m} / \mathrm{z}=18$ and 20) of about 1.3:1 (see Scheme 1). It is evident from this that deconvolution of the mass spectra is needed to estimate the individual amounts of $\mathrm{H}_{2} \mathrm{O}, \mathrm{HOD}$, and $\mathrm{D}_{2} \mathrm{O}$ from the composite mass spectra. To do this, however, one needs to obtain the reference mass spectrum of pure HOD. This is not possible since HOD (or any other mixed isotopologue that exchanges its protons and deuterons) cannot exist in the absence of $\mathrm{H}_{2} \mathrm{O}$ and $\mathrm{D}_{2} \mathrm{O}$ due to the

Published online May 19, 2006

Address reprint requests to Dr. Z. Mester, Institute for National Measurement Standards, National Research Council Canada, 1200 Montreal Rd., Ontario K1A 0R6, Canada. E-mail: zoltan.mester@nrc.ca rapid isotope-exchange equilibrium $2 \mathrm{HOD} \rightleftharpoons \mathrm{H}_{2} \mathrm{O}+$ $\mathrm{D}_{2} \mathrm{O}$.

Using the best available commercial high-resolution mass spectrometers, one can possibly address the $\mathrm{H}_{2} \mathrm{O}$ / $\mathrm{HOD} / \mathrm{D}_{2} \mathrm{O}$ system (requiring $m / \Delta m>12,000$ to fully resolve isobars $\mathrm{OD}^{+}$and $\mathrm{H}_{2} \mathrm{O}^{+}$); however, to do the same for heavier element hydrides, such as HDSe or $\mathrm{AsH}_{2} \mathrm{D}$, mass resolving power of at least 50,000 is required. Such resolution currently is attainable only for GC-FTMS instrumentation. Gas-phase IR spectroscopy could be an alternative option to the above mentioned problem.

While introduction of deuterium-labeled reagents potentially offers new information, such as quantitation or reaction mechanism elucidation, the interpretation of experimental data becomes cumbersome due to the possible spectral overlaps. The aim of this study is to outline mathematical approaches to solve these problems: (1) extraction/reconstruction of pure isotopologue mass spectra; (2) estimation of isotopologue concentration profiles from the mass spectra of their mixtures.

\section{Experimental}

\section{Chemicals}

The following reagents were used: $\mathrm{NaBH}_{4}$ pellets (Alfa Aesar, Ward Hill, MA); $\mathrm{NaBD}_{4}$ pellets $(99 \%$ D, Cambridge Isotope Laboratories, Andover, MA); 37\%, DCl in $\mathrm{D}_{2} \mathrm{O}(99.5 \% \mathrm{D}$, Aldrich, St. Louis, $\mathrm{MO}) ; 30 \% \mathrm{NaOD}$ in $\mathrm{D}_{2} \mathrm{O}\left(99 \% \mathrm{D}\right.$, Aldrich) and $\mathrm{D}_{2} \mathrm{O}(99 \% \mathrm{D}$, Aldrich). A solution of $\mathrm{NaBH}_{4}$ prepared in $\mathrm{H}_{2} \mathrm{O}$ was stabilized by 


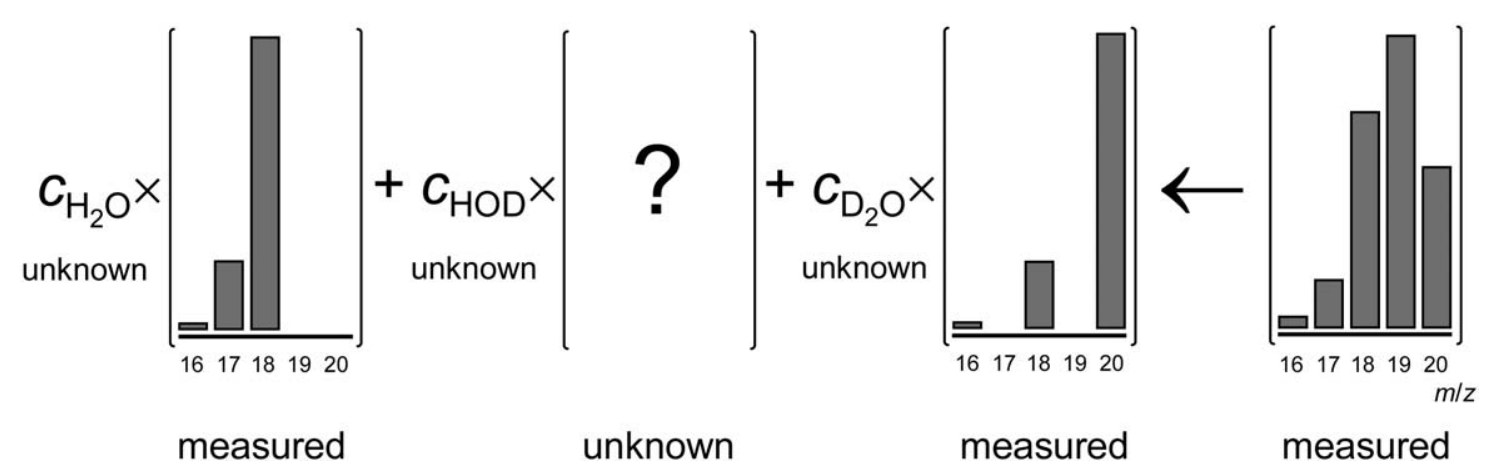

Scheme 1. Statement of the problem: Amounts of individual water isotopologues cannot be estimated from their EI mass spectra without deconvolution.

adding $\mathrm{NaOH}$ up to $0.1 \mathrm{M}$ final concentration. A solution of $\mathrm{NaBD}_{4}(0.25 \mathrm{M})$, prepared in $\mathrm{D}_{2} \mathrm{O}$ was stabilized by adding $\mathrm{NaOD}$ up to $0.1 \mathrm{M}$ final concentration.

An enriched isotopic standard solution of $260 \mu \mathrm{g}$ $\mathrm{mL}^{-1}{ }^{82} \mathrm{Se}(\mathrm{IV})$ in $\mathrm{HNO}_{3}$ aqueous media (Oak Ridge National Laboratory, Oak Ridge, TN) was used to spike the reaction media in septum-sealed vials for cold vapor generation of their corresponding hydrides. All other reagents were of analytical grade.

\section{Apparatus}

A Hewlett-Packard 6890 gas chromatograph (Wilmington, DE) operated in the splitless mode and equipped with a Hewlett-Packard 5973 mass selective detector was fitted with a DB-1 capillary column (30 $\mathrm{m} \times 0.25 \mathrm{~mm}$ i.d. $\times 1 \mu \mathrm{m}$; Vallobond VB-1). A $5 \mathrm{~mL}$ gas-tight syringe (Hamilton, Reno, NV) was employed for sampling headspace gases from reaction vials.

Screw cap reaction vials fitted with PTFE/silicone septa (5-10 mL, Pierce Chemical Co., Rockford, IL) were used according to experimental requirements. The GC was operated under the following conditions: injector temperature $150{ }^{\circ} \mathrm{C}$; oven temperature $35^{\circ} \mathrm{C}$ (isothermal). The carrier gas was He at $1.2 \mathrm{~mL} \mathrm{~min}^{-1}$.

$\mathrm{H}_{2}$ Se generation. The reaction vial $(10 \mathrm{~mL})$ containing 2 $\mathrm{mL}$ of $1 \mathrm{M} \mathrm{HCl}$, about $10 \mu \mathrm{g}$ of ${ }^{82} \mathrm{Se}(\mathrm{IV})$ and a Teflon coated stir-bar was capped and two stainless steel needles were inserted into the septum. Vigorous stirring of the solution was started and nitrogen was then introduced through one needle to purge atmospheric oxygen from the headspace of the vial. The two needles were then removed and $1 \mathrm{~mL}$ of $0.25 \mathrm{M} \mathrm{NaBH}_{4}$ solution was injected using a plastic syringe fitted with a stainless steel needle. Headspace gases $(2-3 \mathrm{~mL})$ were subsequently sampled with a gas tight syringe and injected into the GC/MS. Mass spectrum of pure $\mathrm{H}_{2} \mathrm{Se}$ was obtained.
$\mathrm{D}_{2} \mathrm{Se}$ generation. Generation of pure $\mathrm{D}_{2} \mathrm{Se}$ was attempted using fully deuterated reaction media. A procedure similar to that described for $\mathrm{H}_{2} \mathrm{Se}$ generation was adopted with the following modifications. In a 5 $\mathrm{mL}$ vial containing $0.5 \mathrm{~mL}$ of $1 \mathrm{M} \mathrm{DCl}$ spiked with 0.05 $\mathrm{mL}$ of ${ }^{82} \mathrm{Se}(\mathrm{IV})$ aqueous standard solution, atmospheric oxygen was degassed and $0.5 \mathrm{~mL}$ of $0.25 \mathrm{M} \mathrm{NaBD}_{4}$ solution was injected.

H/D exchange experiments. Pure $\mathrm{H}_{2} \mathrm{Se}$ was prepared as described above. Two aliquots of the $\mathrm{H}_{2} \mathrm{Se}$ headspace gas (each of 3-5 mL volume) were collected in a rapid sequence. The first aliquot was injected into a $5 \mathrm{~mL}$ reaction vial (the exchange vial) containing $1 \mathrm{~mL}$ of 0 , 3 and $6 \mathrm{M} \mathrm{DCl}$ in $\mathrm{D}_{2} \mathrm{O}$ and continuously shaken throughout the experiment. The second $\mathrm{H}_{2} \mathrm{Se}$ headspace aliquot was injected into the GC/MS to verify the isotopic composition of the synthesized $\mathrm{H}_{2} \mathrm{Se}$. The isotopic composition of the injected hydride in the headspace of the exchange vial was checked at regular intervals by GC/MS. Previous experiments in which $\mathrm{H}_{2} \mathrm{Se}$ was injected onto a column pretreated with $\mathrm{DCl}$ vapors have demonstrated that the $\mathrm{H} / \mathrm{D}$ exchange does not take place in the GC capillary column [1].

\section{Results and Discussion}

The first aim of the study is the extraction of pure component mass spectra from their mixtures. In mathematical terms, this is an inverse problem of spectral reconstruction: given composite spectra, extract the individual component mass spectra and their concentration. In information theory, this is referred to as the blind source separation and independent component analysis. The $\mathrm{H}_{2} \mathrm{O} / \mathrm{HOD} / \mathrm{D}_{2} \mathrm{O}$ system resembles a so-called gray system because we have partial knowledge of the spectra of the components involved. Also, the number of components is known. 


\section{Reconstruction of HOD Mass Spectrum}

Alternating least-squares model. Any mass spectra of the $\mathrm{H}_{2} \mathrm{O}, \mathrm{HOD}$, and $\mathrm{D}_{2} \mathrm{O}$ mixture can be represented in a general equation:

$\left[\begin{array}{c}I_{\mathrm{mlz}}^{\mathrm{mix}} \\ \ldots \\ I_{\mathrm{mlz} 20}^{\mathrm{mix}}\end{array}\right]=\mathrm{a}_{\mathrm{H}_{2} \mathrm{O}}\left[\begin{array}{c}I_{16}^{\mathrm{H}_{2} \mathrm{O}} \\ \ldots \\ I_{20}^{\mathrm{H}_{2} \mathrm{O}}\end{array}\right]+\mathrm{a}_{\mathrm{HOD}}\left[\begin{array}{c}I_{16}^{\mathrm{HOD}} \\ \ldots \\ I_{20}^{\mathrm{HOD}}\end{array}\right]+\mathrm{a}_{\mathrm{D}_{2} \mathrm{O}}\left[\begin{array}{c}I_{16}^{\mathrm{D}_{2} \mathrm{O}} \\ \ldots \\ I_{20}^{\mathrm{D}_{2} \mathrm{O}}\end{array}\right]$

This demonstrates that the observed [normalized] mass spectrum is a weighted sum of all the isotopomer mass spectra. Weighting factors, $a$, are the amount fractions of each isotopomer in the mixture. Eq 1 can be rewritten as $I=F \times a+\varepsilon$, where $I_{5 \times 1}$ is the observed mass spectrum vector of the mixture ( 5 is the number of $m / z$ channels), $F_{5 \times 3}$ is the matrix of three individual component mass spectra (stacked together), $a_{3 \times 1}$ is their abundance (amount fraction) vector and $\varepsilon_{5 \times 1}$ is the instrumental noise vector [2]. In an alternating leastsquares method (also known as iterative least-squares), many possible (alternating) sets of HOD mass spectra are generated, which then are used (one-by-one) to reconstruct the mass spectra of the $\mathrm{H}_{2} \mathrm{O} / \mathrm{HOD} / \mathrm{D}_{2} \mathrm{O}$ mixtures. A large set of possible HOD mass spectra can be obtained using the uniformly distributed random number generator. Three random numbers, $x_{i} \in[0$. . .1], are generated in each set of calculations: $x_{1}, x_{2}$, and $x_{3}$, which correspond to $m / z=16,17$, and 18 intensities (normalized) in the HOD mass spectrum; $m / z=19$ intensity, $x_{4}=1-x_{1}-x_{2}-x_{3}$. If $x_{4}<0$, another set of random numbers is generated until $x_{4}>0$. Each of the generated (non-negative) HOD spectra is then used in eq 1 to reconstruct a given experimental mass spectrum of the $\mathrm{H}_{2} \mathrm{O} / \mathrm{HOD} / \mathrm{D}_{2} \mathrm{O}$ mixture using the least-squares optimization. A HOD mass spectrum that fits the experimental mass spectra with the smallest residual error is the result of the least square optimization

$$
\begin{aligned}
& \varepsilon=I-F \times a \\
& \min (G)=\varepsilon^{T} \varepsilon
\end{aligned}
$$

Here, a global minimum, $\min (G)$, of the variance of the residual errors is sought. Alternating least-squares have been used previously in spectroscopy to reconstruct the infrared spectra of HOD and eventually determine the individual amounts of $\mathrm{H}_{2} \mathrm{O}, \mathrm{HOD}$, and $\mathrm{D}_{2} \mathrm{O}$ in the $\mathrm{H}_{2} \mathrm{O} / \mathrm{D}_{2} \mathrm{O}$ mixtures $[3,4]$. This technique is also of general interest in solving liquid chromatography mass spectrometry co-elution problems [5].

In the case of the $\mathrm{H}_{2} \mathrm{O} / \mathrm{D}_{2} \mathrm{O}$ system, the mass spectra of $\mathrm{H}_{2} \mathrm{O}, \mathrm{D}_{2} \mathrm{O}$, and that of the resulting mixture are known. The remaining five independent variables, $a\left(\mathrm{H}_{2} \mathrm{O}\right), a(\mathrm{HOD})$, and the intensities of $m / z=16,17$, and 18 in the mass spectrum of HOD, are unknown. Other variables: $a\left(\mathrm{D}_{2} \mathrm{O}\right)$ and $I(\mathrm{HOD}, m / z 19)$, are dependent since both concentrations and mass spectra are normalized to unity. As well, $I(\mathrm{HOD}, m / z 20)=0$. Note that the system is under-determined by one dimension (four independent measurements and five unknown variables) and, therefore, it is impossible to obtain an analytical solution of the HOD mass spectrum. Instead, we can obtain the range of solutions that are consistent with certain assumptions discussed below.

Since the system described above is under-determined, contextual information has to be supplied. Such information can be the expected intensity of the HOD molecular ion. The normalized molecular ion intensities for $\mathrm{H}_{2} \mathrm{O}$ and $\mathrm{D}_{2} \mathrm{O}$ are $0.799 \pm 0.001( \pm 2 s)$ and $0.803 \pm$ 0.001 , respectively; therefore it is reasonable to assume that the intensity of the HOD molecular ion is expected to be within that range. Note that the real isotope dilution analytical protocols operate on the very same basic assumption. The reconstructed mass spectrum of HOD using the alternating least-squares algorithm with the above mentioned restriction (assumption) is shown in Figure 1.

Entropy minimization model. Mass spectra of pure components can be reconstructed using entropy minimization as recently outlined by Zhang et al. [6]. In essence, this approach is a pattern discovery (recognition) via entropy minimization. The objective function to be minimized thus is the entropy of the extracted mass spectrum. In information theory, entropy is a measure of the average amount of information required to describe the distribution of some variable of interest. The most commonly used measure of entropy was introduced by Shannon $(S=p \cdot \ln p)$ [7]. This classical entropy definition was previously used in reconstruction of continuous NMR, Raman, and IR spectra where $p$ has been quantitatively linked with the first or higher order derivatives [8-12]. However, such an entropy definition (smoothness of the spectra) cannot be transferred in the context of mass spectrometry where the individual masses are discrete by their nature and, thus, a modified non-logarithmic entropy definition is used in this model recently developed by the Garland group [6, 12].

An overall weighted two band-target entropy minimization algorithm can be outlined in the following steps. First, a mass spectral data matrix $I_{k \times n}$ is compiled from $k$ mass spectra each having $n$ mass-to-charge channels ( $k \geq$ number of possible isotopologues). Each mass spectrum is normalized to unity total ion intensity. Second, a singular value decomposition of $I_{k \times n}$ matrix is performed [13]. This procedure represents any $k \times n$ matrix as a product of three matrices $\left(U_{k \times n}\right.$ and $V_{n \times n}$ are orthonormal, e.g., $U^{T} U=V^{T} V=1$ ):

$$
I_{k \times n}=U_{k \times n} \times \sum_{n \times n} \times V_{n \times n}^{T}
$$

This leads to the diagonal singular value matrix $\Sigma$ and the right singular value matrix $V^{T}$. The obtained singular value decomposition is used further for noise reduction [14]. The main idea is that the experimental $I_{k \times n}$ matrix contains components of large and low variation. It can be shown that the small singular values 

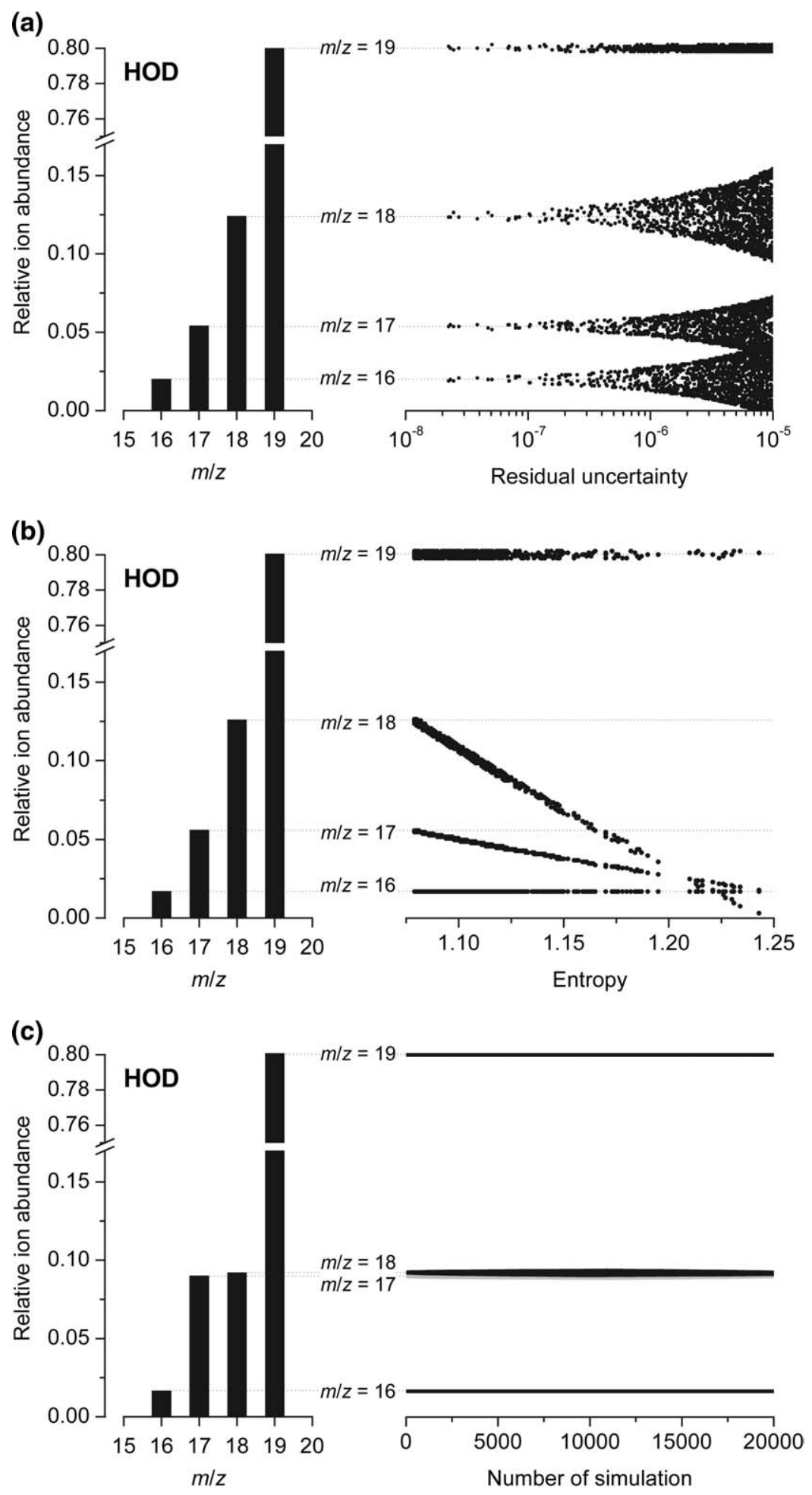

Figure 1. Construction of HOD mass spectrum using three conceptually different approaches: alternating least-squares (a), weighted two band-target entropy minimization (b), and statistical mass balance model (c).

in $\Sigma$ mainly represent the noise. This noise is eliminated by keeping only the first $j$ non-zero singular values $(j \leq$ $k$ ) [15]. It is worth mentioning that the $\Sigma$ matrix range (which equals $j$ ) represents the number of individual compounds whose spectra are to be deconvoluted. The filtered (truncated) representation of spectral matrix $I_{j \times n}$ now becomes

$$
I_{j \times n}=U_{j \times j} \times \sum_{j \times j} \times V_{j \times n}^{T}
$$


The pure component mass spectra can be obtained using the transformation matrix $T$ :

$$
A_{1 \times n}=T_{1 \times j} \times\left(\sum_{j \times j} \times V_{j \times n}^{T}\right)
$$

The presence of matrix $\Sigma$ in this equation serves as a weighting procedure, making the model more robust. Mass spectral reconstruction of $j$ independent components now becomes a problem of finding all $T$ vectors. There are certain restrictions any candidate $T$ vector must comply with. First, it has to produce a nonnegative pure component spectrum estimate $A_{1 \times n}$. Choosing the two target bands ( $\mathrm{m} / \mathrm{z}$ channels) $A_{x}$ and $A_{y}$ is the crucial step of the method. During this step $A_{1 \times n}$ matrix is normalized with respect to the total intensity of the target bands:

$$
A_{1 \times n}^{*}=\frac{A_{1 \times n}}{A_{x}+A_{y}}
$$

Depending on the targeted $\mathrm{m} / \mathrm{z}$ channels, mass spectra of different isotopologues are extracted. For example, targeting the $\mathrm{m} / \mathrm{z}=18$ and 20 yields a mass spectrum of $\mathrm{D}_{2} \mathrm{O}$, targeting $\mathrm{m} / \mathrm{z}=17$ and 18 yields a $\mathrm{H}_{2} \mathrm{O}$ mass spectrum, and targeting $\mathrm{m} / \mathrm{z}=17$ and 19 or 18 and 19 results in retrieval of the HOD spectrum. Second, component contribution (concentration) in each of the $k$ mass spectra also has to be non-negative:

$$
c_{k \times 1}=I_{k \times n} \times A_{n \times 1}^{* T} \times\left(A_{1 \times n}^{*} \times A_{n \times 1}^{* T}\right)^{-1}
$$

The objective function (entropy) to be minimized is simply the sum of the channel intensities in the reconstructed mass spectrum:

$$
\min (G)=\sum_{n} A_{1 \times n}^{*}
$$

Minimization of the objective function (in terms of $T$ vector) is usually achieved using the simulated annealing algorithm, which is a good choice for a global minimum search in many dimensions $[16,17]$. In our situation, the number of dimensions is small (three for the $\mathrm{H}_{2} \mathrm{O} / \mathrm{HOD} / \mathrm{D}_{2} \mathrm{O}$ system); therefore we use the exhaustive random search.

As mentioned above, the model seeks the lowest entropy (most simple) mass spectra within the nonnegativity constraints. In a particular $\mathrm{H}_{2} \mathrm{O} / \mathrm{HOD} / \mathrm{D}_{2} \mathrm{O}$ example, the three component concentration and mass spectral information is condensed into five spectral channels; therefore the system is undetermined and an infinite number of HOD mass spectra can be obtained. Since one of such possible solutions is a HOD spectrum with no fragment ions, this is the obvious minimum entropy result (the simplest mass spectrum). In other words, additional contextual information is needed to separate the mathematical solution from the chemically meaningful solution of the HOD mass spectrum. The same applies to the reconstruction of the HDSe mass spectrum. The blind deconvolution lowest entropy result for the HOD mass spectrum is $(0.000 ; 0.000 ; 0.000$; $1.000 ; 0.000)$. This result clearly has no chemical meaning, especially considering the experimentally measured mass spectra of $\mathrm{H}_{2} \mathrm{O}$ and $\mathrm{D}_{2} \mathrm{O}$. When the abundance of the HOD molecular ion is set within the range of $\mathrm{H}_{2} \mathrm{O}$ and $\mathrm{D}_{2} \mathrm{O}$ molecular ions, the reconstructed mass spectrum of HOD using the weighted two-band target entropy minimization algorithm (targeting $\mathrm{m} / \mathrm{z}=18$ and 19) with the above mentioned restriction of molecular ion intensity is $(0.017 ; 0.056 ; 0.126 ; 0.801 ; 0.000)$ as shown in Figure 1. Although these two results are quantitatively different, nevertheless, they are nearly identical when compared using the spectral contrast angle ( $99 \%$ similarity). Clearly, the spectral contrast angle is not a good choice for comparing the experimental and the reconstructed spectra due to its insensitivity to low intensity ions. This was also recently pointed out by Zhang who proposed using the ratio of the geometric and the arithmetic means as a similarity measure between the mass spectra [18]. Using this criterion, the similarity between constrained and unconstrained HOD spectra becomes $89 \%$. In the first unconstrained band-target entropy minimization reconstruction of mass spectra, the Garland group achieves $76-92 \%$ similarity (calculated using the ratio of geometric and arithmetic means) between the reference and measured spectra in a four component mixture (ethanol, acetone, hexane, and toluene in the $\mathrm{m} / \mathrm{z}=10-100$ range) [6].

Clearly, when dealing with under-determined overlapping isotopologue systems, ion intensities in the extracted pure component mass spectra can be biased if no contextual feedback is provided. As we have seen, although such bias might be of little importance for spectral recognition, it is, nevertheless, significant for quantitative purposes.

Statistical model. To predict mass spectra of isotopologues one needs to consider two factors: differences in symmetry numbers for particular fragmentation pathways (e.g., loss of $\mathrm{H}$ from $\mathrm{H}_{2} \mathrm{O}$ versus $\mathrm{HOD}$ ) and possible rate constant differences between the two isotopic pathways (loss of $\mathrm{H}$ versus loss of $\mathrm{D}$ from the same ion) [19]. All the possible fragmentation reaction pathways can be written for $\mathrm{H}_{2} \mathrm{O}, \mathrm{HOD}$, and $\mathrm{D}_{2} \mathrm{O}$, and for each of the reactions we can assign a probability coefficient that accounts for the mass balance between the precursor and fragment ions including the statistical factor as shown in Scheme 2. This model assumes that the probability of a certain ligand is directly proportional to its amount in the precursor ion. In other words, the loss of an $\mathrm{H}^{-}$from $\mathrm{H}_{2} \mathrm{O}^{+}$is assumed to be twice as probable as from $\mathrm{HOD}^{+}$. Auto-ionization (formation of $\mathrm{H}_{3} \mathrm{O}^{+}, \mathrm{D}_{3} \mathrm{O}^{+}, \mathrm{H}_{2} \mathrm{DO}^{+}$, and $\mathrm{HD}_{2} \mathrm{O}^{+}$) is excluded from this scheme due to its small contribution $(<1 \%)$. These mathematical coefficients are obtained from the experimental mass spectra of pure $\mathrm{H}_{2} \mathrm{O}$ and $\mathrm{D}_{2} \mathrm{O}$ and then are used to reconstruct the mass spectrum of $\mathrm{HOD}$ as 

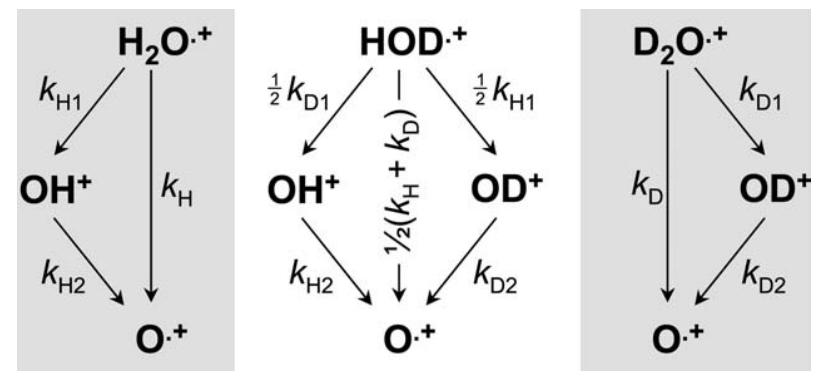

Scheme 2. Statistical mass balance model of the $\mathrm{H}_{2} \mathrm{O}$ and $\mathrm{D}_{2} \mathrm{O}$ mass spectra used to reconstruct the mass spectrum of the HOD. Coefficients $k$ represent the reaction probabilities.

shown in Scheme 2. In this model, the (normalized) intensity of $m / z 17$ for $\mathrm{H}_{2} \mathrm{O}$ is $k_{\mathrm{H} 1}\left(1-k_{\mathrm{H} 2}\right)$ and $0.5 k_{\mathrm{D} 1}(1$ $-k_{\mathrm{H} 2}$ ) for HOD.

One can immediately see from this scheme, that the system is (again) under-determined: with three independent variables $\left(k_{\mathrm{H} 1}, k_{\mathrm{H} 2}\right.$, and $\left.k_{\mathrm{H}}\right)$ and only two independent measurements, the intensities of $\mathrm{H}_{2} \mathrm{O}^{+}$ $(\mathrm{m} / \mathrm{z} 18)$ and $\mathrm{OH}^{+}(\mathrm{m} / \mathrm{z} 17)$, since the intensity of the remaining ion $\left(\mathrm{O}^{+}\right)$in a normalized mass spectra is bound to the sum of all the other relative ion intensities. The same applies for $\mathrm{D}_{2} \mathrm{O}$ coefficients. In other words, one can have an infinite number of solutions to the model described above. Despite this, we can assign all the possible values of $k_{\mathrm{H} 1}$ and $k_{\mathrm{D} 1}\left(k_{\mathrm{H} 1}\right.$ and $\left.k_{\mathrm{D} 1} \in[0 \ldots 1]\right)$ and for each of these values $\left(k_{\mathrm{H} 2}, k_{\mathrm{H}}\right)$ and $\left(k_{\mathrm{D} 2}, k_{\mathrm{D}}\right)$ are calculated. Any set of $k_{\mathrm{H} 1}, k_{\mathrm{H} 2}$, and $k_{\mathrm{H}}$ has to fit the experimental mass spectrum of $\mathrm{H}_{2} \mathrm{O}$ within the specified error threshold. The same applies for $\mathrm{D}_{2} \mathrm{O}$. Also, any negative values of $k$ are eliminated from the solution set. This procedure is essentially a non-negative least-squares optimization [20]. Once all the possible sets of $\left(k_{\mathrm{H} 1}, k_{\mathrm{H} 2}, k_{\mathrm{H}}\right)$ and $\left(k_{\mathrm{D} 1}, k_{\mathrm{D} 2}, k_{\mathrm{D}}\right)$ are identified, mass spectra of HOD are then obtained for every possible combination between those two sets according to the Scheme 2 . Interestingly enough, the obtained ion abundances in mass spectra of HOD are insensitive to the particular values of $k$ used. This is because the ion intensities in the HOD mass spectrum are functions of the ratios between the $k_{\mathrm{H} i}$ and $k_{\mathrm{D} i}$ :

$$
\begin{aligned}
& I_{m / z 19}=0.5 I\left(\mathrm{H}_{2} \mathrm{O}, m / z 18\right)+0.5 I\left(\mathrm{D}_{2} \mathrm{O}, m / z 20\right) \\
& I_{m / z 18}=0.5 I\left(\mathrm{H}_{2} \mathrm{O}, m / z 17\right) \cdot\left(1-k_{\mathrm{D} 2}\right) /\left(1-k_{\mathrm{H} 2}\right) \\
& I_{m / z 17}=0.5 I\left(\mathrm{D}_{2} \mathrm{O}, m / z 18\right)\left(1-k_{\mathrm{H} 2}\right) /\left(1-k_{\mathrm{D} 2}\right)
\end{aligned}
$$

Even though the complete mass spectrum of HOD $(m / z \in 16 \ldots 19)$ cannot be experimentally obtained, the ratio of $\mathrm{HOD}^{+}$to $\mathrm{OH}^{+}$can be measured from a mixture of $\mathrm{D}_{2} \mathrm{O}$ with a small amount $(<5 \%)$ of $\mathrm{H}_{2} \mathrm{O}$. In such mixtures virtually all of the ${ }^{1} \mathrm{H}$ isotopes are in the form of HOD. Besides that, odd $\mathrm{m} / \mathrm{z}$ values have zero intensity in the $\mathrm{D}_{2} \mathrm{O}$ mass spectrum. Hence, the ratio of $\mathrm{m} / \mathrm{z}$ $19 / 17$ in such $\mathrm{D}_{2} \mathrm{O} / \mathrm{H}_{2} \mathrm{O}$ mixtures is a direct estimation of a partial HOD mass spectrum. Since the intensity of $\mathrm{m} / \mathrm{z} 17$ is low, the uncertainty of the experimental 19/17 ratio is rather large. Nevertheless, the average experimental value of $14 \pm 6( \pm 2 s)$ is comparable to the alternating least-squares estimate of $16 \pm 3$, the entropy minimization estimate of $14.5 \pm 0.3$ and the statistical mass-balance estimate of $8.9 \pm 0.1$.

Alternating least-squares and entropy minimization yield virtually identical mass spectra of HOD. Although the $\mathrm{m} / \mathrm{z} 17$ and 18 ion intensities in the HOD mass spectrum as obtained from the statistical model appears qualitatively very different from the other two estimates, the spectral similarity of the HOD mass spectra among all three models exceeds $99.8 \%$ (calculated either as the spectral contrast angle between the unity length normalized spectra [21] or as the ratio of the geometric and arithmetic mean between them [18]). For reference purposes, all the mass spectra are tabulated in Table 1.

\section{Reconstruction of HDSe Mass Spectrum}

Reconstruction of selenium hydride mass spectra is a problem similar to that of the water example. The difficulty of the pure component spectra reconstruction, however, is increased because the $\mathrm{D}_{2} \mathrm{Se}$ mass spectrum is difficult to obtain even by reaction of trace amounts of Se(IV) with $\mathrm{NaBD}_{4}$ in deuterium-only medium (DCl $+\mathrm{NaBD}_{4}, x(\mathrm{D})>97 \%$ ) as discussed previously in the Experimental section.

Accordingly, alternating least-squares cannot be used to reconstruct the mass spectrum of HDSe since no experimental estimate of $\mathrm{D}_{2} \mathrm{Se}$ is available. In spite of this, Figure 2 shows the entropy minimization estimates of $\mathrm{H}_{2} \mathrm{Se}$, HDSe, and $\mathrm{D}_{2} \mathrm{Se}$ mass spectra (containing only ${ }^{82} \mathrm{Se}$ isotope). The $\mathrm{H}_{2} \mathrm{Se}$ mass spectrum was recovered by targeting the $m / z=82$ and 84 , HDSe by targeting $\mathrm{m} / \mathrm{z}$ $=82$ and 85 , and the $\mathrm{D}_{2}$ Se mass spectrum was recovered by targeting the $m / z=82$ and 86 . To reduce the bias in recovered ion intensities, the molecular ion in the HDSe mass spectrum was forced to be in the range of $\mathrm{H}_{2} \mathrm{Se}$ and $\mathrm{D}_{2} \mathrm{Se}$ molecular ion intensities. One can see that the reconstructed $\mathrm{H}_{2} \mathrm{Se}$ mass spectrum agrees well with the experimental measurements (Figure 2). The

Table 1. Mass spectra of $\mathrm{H}_{2} \mathrm{O} / \mathrm{HOD} / \mathrm{D}_{2} \mathrm{O}$ and $\mathrm{H}_{2} \mathrm{Se}$ /HDSe/ $\mathrm{D}_{2} \mathrm{Se}$

\begin{tabular}{rccc}
\hline & $\mathrm{H}_{2} \mathrm{O}^{\mathrm{a}}$ & $\mathrm{HOD}^{\mathrm{b}, \mathrm{c}, \mathrm{d}}$ & $\mathrm{D}_{2} \mathrm{O}^{\mathrm{a}}$ \\
\hline \hline $\mathrm{m} / \mathrm{z} 16$ & $0.0161_{5}$ & $0.017|0.017| 0.020$ & $0.0170_{2}$ \\
17 & $0.1852_{7}$ & $0.090|0.056| 0.054$ & \\
18 & $0.7988_{6}$ & $0.092|0.126| 0.124$ & $0.1803_{5}$ \\
19 & & $0.801|0.801| 0.800$ & \\
20 & & & $0.8027_{6}$ \\
\hline & $\mathrm{H}_{2}{ }^{82} \mathrm{Se}^{\mathrm{a}}$ & $\mathrm{HD}^{82} \mathrm{Se}^{\mathrm{b}, \mathrm{c}}$ & $\mathrm{D}_{2}{ }^{82} \mathrm{Se}^{\mathrm{c}}$ \\
\hline \hline $\mathrm{m} / \mathrm{z} 82$ & 0.375 & $0.368 \mid 0.348$ & 0.346 \\
83 & 0.176 & $0.087 \mid 0.073$ & 0.000 \\
84 & 0.449 & $0.087 \mid 0.093$ & 0.166 \\
85 & & $0.457 \mid 0.486$ & 0.000 \\
86 & & & 0.488 \\
\hline
\end{tabular}

${ }^{a}$ Experimental mass spectra. $0.0161_{5}$ means $0.0161 \pm 0.0005(2 s)$ bStatistical mass balance model

'Weighted two band-target entropy minimization

dAlternating least squares reconstruction 


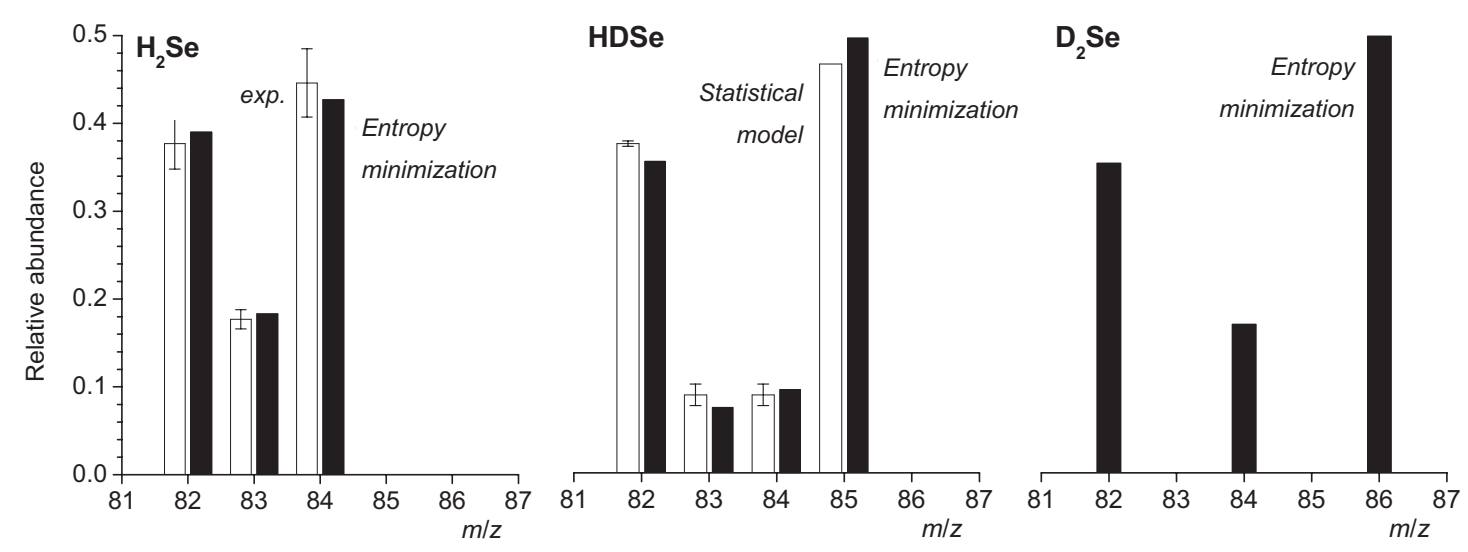

Figure 2. Reconstructed mass spectra of $\mathrm{H}_{2}{ }^{82} \mathrm{Se}, \mathrm{HD}^{82} \mathrm{Se}$, and $\mathrm{D}_{2}{ }^{82} \mathrm{Se}$ using band-target entropy minimization and statistical mass balance model.

HDSe mass spectrum was also modeled using the statistical mass balance model assuming that the ion intensities in the $\mathrm{D}_{2} \mathrm{Se}$ mass spectrum are the same as those in $\mathrm{H}_{2} \mathrm{Se}$ spectrum. Using these assumptions, the obtained HDSe mass spectrum agrees rather well with the lowest entropy estimate (Figure 2). For reference purposes, all the mass spectra are tabulated in Table 1.

\section{Quantitation of Isotopologues}

\section{Using the Reconstructed Mass Spectra}

$\mathrm{H}_{2} \mathrm{O} / \mathrm{HOD} / \mathrm{D}_{2} \mathrm{O}$. The extracted HOD mass spectra using alternating least-squares or entropy minimization approaches are identical (see Figure 1) and, therefore, the deconvoluted isotopologue concentrations will be the same using the two models. In contrast to this, the average $m / z=17$ and 18 ion intensities in the HOD mass spectrum obtained from the statistical models are different (see Figure 1); nevertheless, the spectral similarity [18] or spectral contrast angle [22] between the three extracted HOD mass spectra is $>99 \%$. The HOD mass spectra (as obtained from statistical and alternating least-squares models) are then used for determination of $\mathrm{H}_{2} \mathrm{O}, \mathrm{HOD}$, and $\mathrm{D}_{2} \mathrm{O}$ amount fractions in various $\mathrm{H}_{2} \mathrm{O} / \mathrm{D}_{2} \mathrm{O}$ mixtures with varying amount fraction of deuterium, $x(\mathrm{D})$, using the linear least-squares isotope pattern reconstruction (Figure 3), [2]. The accuracy of the deconvoluted isotopologue concentrations was verified using the gravimetrically prepared mixtures of $\mathrm{H}_{2} \mathrm{O}$ and $\mathrm{D}_{2} \mathrm{O}$ with known $\mathrm{D} / \mathrm{H}$ ratios. A total $\mathrm{H} / \mathrm{D}$ amount ratio between the two gravimetrically prepared $\mathrm{H}_{2} \mathrm{O} / \mathrm{D}_{2} \mathrm{O}$ mixtures with $n(\mathrm{H}) / n(\mathrm{D})=2.005$ was determined as $2.03 \pm 0.02( \pm 2 s, n=10)$ and $2.02 \pm 0.02( \pm 2 s$, $n=10)$ using alternating least-squares and statistical models, respectively. Also, the $\mathrm{H}_{2} \mathrm{O} / \mathrm{HOD} / \mathrm{D}_{2} \mathrm{O}$ concentration profiles are in good agreement with earlier studies (see Figure 4 in Libnau et al. [3]).

Mixtures of $\mathrm{H}_{2} \mathrm{O}$ and $\mathrm{D}_{2} \mathrm{O}$ are characterized by the equilibrium $\mathrm{H}_{2} \mathrm{O}+\mathrm{D}_{2} \mathrm{O} \rightleftharpoons 2 \mathrm{HOD}$ and isotopic selfexchange reaction of water in gaseous or liquid phases has been the subject of numerous investigations and debates over the past decades [24, 25]. Although seemingly simple system, estimates of the $\mathrm{H}_{2} \mathrm{O} / \mathrm{D}_{2} \mathrm{O}$ isotope exchange equilibrium constant (in both gaseous and liquid phases) range between 3.41 and the geometric mean of $4.0[3,26]$. This value has been recently (re)estimated using IR and NMR techniques [3, 27].

Using the above described HOD reconstruction/ deconvolution algorithm, we can easily calculate the equilibrium constant from the amount fractions of $\mathrm{H}_{2} \mathrm{O}$, $\mathrm{HOD}$, and $\mathrm{D}_{2} \mathrm{O}$. Replicate measurements in the range of $x(\mathrm{D}) \approx 50-70 \%$ lead to the average value of $K=3.85 \pm$ $0.03( \pm 2 s)$ using the alternating least-squares deconvolution and entropy minimization model. $K$ values obtained from the statistical mass balance model were by $\sim 7 \%$ lower. This in good agreement with the "best" theoretical value of 3.85 [23]. The two most recent liquid phase equilibrium constant measurements (at $298 \mathrm{~K}$ ) are $3.86 \pm 0.07$ [3] and $3.86 \pm 0.01$ [27]. Note that the

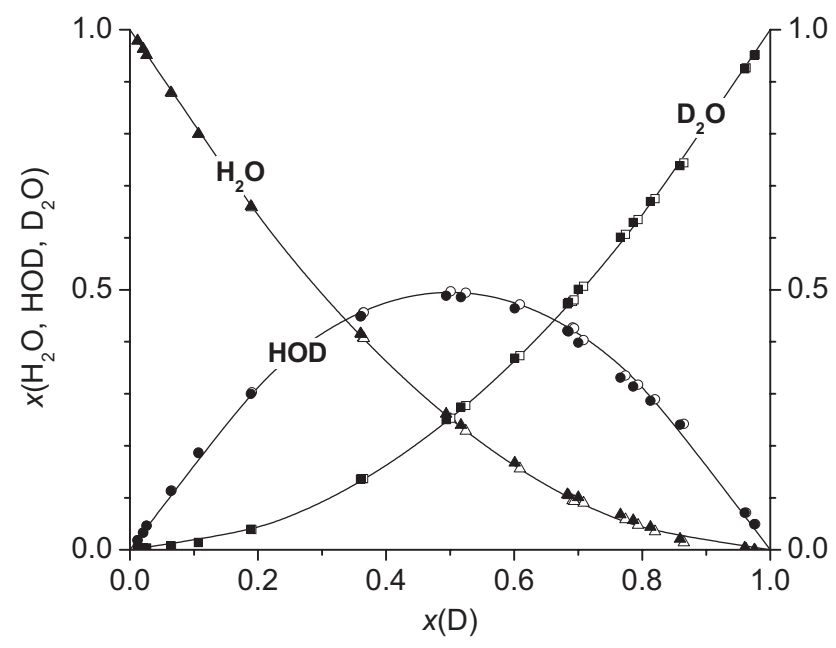

Figure 3. Amount fraction profiles for $\mathrm{H}_{2} \mathrm{O}, \mathrm{HOD}$, and $\mathrm{D}_{2} \mathrm{O}$ in $\mathrm{H}_{2} \mathrm{O} / \mathrm{D}_{2} \mathrm{O}$ mixtures as a function of the deuterium amount fraction in the solution. Open dots are from the alternating leastsquares algorithm and filled dots are from the mass balance model. Lines correspond to the "best" theoretical equilibrium constant $K=3.85$ by Wolfsberg et al. [23] 
Table 2. Comparison of the three models used for the reconstruction of HOD and HDSe mass spectra

\begin{tabular}{lcc}
\hline Reconstruction model & Input information & Assumptions \\
\hline \hline Alternating least squares & $\begin{array}{c}\text { Mass spectra of pure } \mathrm{H}_{2} \mathrm{O}, \mathrm{D}_{2} \mathrm{O} \text { and } \\
\mathrm{H}_{2} \mathrm{Se}, \mathrm{D}_{2} \mathrm{Se}\end{array}$ & $\begin{array}{c}\text { Range for the possible HDSe or HOD molecular } \\
\text { ion intensity is needed to obtain accurate mass } \\
\text { spectra of HDSe and HOD. }\end{array}$ \\
Entropy minimization & $\begin{array}{c}\text { At least three independent mass } \\
\text { spectra of various } \mathrm{H}_{2} \mathrm{O} / \mathrm{HOD} /\end{array}$ & $\begin{array}{c}\text { Range for the possible } \mathrm{HDSe} \text { and } \mathrm{HOD} \text { molecular } \\
\text { ion intensity is expected to be within the } \\
\text { values from } \mathrm{H}_{2} \mathrm{Se}, \mathrm{D}_{2} \mathrm{Se} \text { and } \mathrm{H}_{2} \mathrm{O}, \mathrm{D}_{2} \mathrm{O} \text { spectra. }\end{array}$ \\
& $\begin{array}{l}\mathrm{D}_{2} \mathrm{O} \text { and } \mathrm{H}_{2} \mathrm{Se} / \mathrm{HDSe} / \mathrm{D}_{2} \mathrm{Se} \\
\text { mixtures. }\end{array}$ & $\begin{array}{c}\text { Probability of ligand loss is directly proportional } \\
\text { to its amount in the precursor ion. }\end{array}$ \\
\hline
\end{tabular}

difference between liquid and gas-phase equilibrium constants is estimated to be only 0.03 [28]. Previous direct mass spectrometric measurements (carried out in the 1960s) have led to the values of $K=3.75 \pm 0.07$ [29] and $3.76 \pm 0.02$ [26]; however, these measurements have been performed at electron accelerating voltages from $3 \mathrm{eV}\left(\mathrm{H}_{2} \mathrm{O}\right)$ [25] to $12-13 \mathrm{eV}\left(\mathrm{H}_{2} \mathrm{Se}\right.$ and $\left.\mathrm{H}_{2} \mathrm{~S}\right)$ [30] to eliminate any fragment formation. Although this classical approach avoids the need for deconvolution, it is clearly not of practical use for a variety of analytical purposes due to the deterioration of method sensitivity and robustness.

$\mathrm{H}_{2} \mathrm{Se} / \mathrm{HDSe} / \mathrm{D}_{2} \mathrm{Se}$. Various gaseous mixtures of $\mathrm{H}_{2} \mathrm{Se} /$ HDSe $/ \mathrm{D}_{2} \mathrm{Se}$ were obtained when $\mathrm{H}_{2}{ }^{82} \mathrm{Se}$ (generated from ${ }^{82} \mathrm{SeO}_{3}{ }^{2-}$ and $2 \mathrm{M} \mathrm{HCl} / \mathrm{NaBH}_{4}$ ) was introduced in the headspace of $\mathrm{D}_{2} \mathrm{O} / \mathrm{DCl}$ environment. In such conditions rapid $\mathrm{H} / \mathrm{D}$ exchange takes place resulting in a rise of $\mathrm{HD}^{82} \mathrm{Se}$ and $\mathrm{D}_{2}{ }^{82} \mathrm{Se}$ concentrations in the headspace. The resulting mixtures were then analyzed using GC/MS, and their composite mass spectra were decomposed using the weighted two band-target entropy minimization algorithm and the statistical model. The relative amount fractions of isotopologues as obtained from both models are in good agreement and are not biased.

Based on reaction purities of reagents, the estimated $\mathrm{H}$ atom fraction in the reaction solution was $<7 \%$. The obtained mixture contained $43 \% \mathrm{H}_{2} \mathrm{Se}$ and $44 \%$ HDSe, and only $13 \% \mathrm{D}_{2} \mathrm{Se}(63 \%$ atom fraction of $\mathrm{H})$. Decreasing the volume of the spike of ${ }^{82} \mathrm{Se}(\mathrm{IV})$ aqueous standard solution to $0.01 \mathrm{~mL}(\mathrm{H}$ atom fraction in reaction solution $<3 \%$ ) improved the purity of the $\mathrm{D}_{2}{ }^{82} \mathrm{Se}$, but it was unsuccessful in the production of pure $\mathrm{D}_{2} \mathrm{Se}$ : the composition of the resulting mixture was $10 \% \mathrm{H}_{2} \mathrm{Se}$, $26 \%$ HDSe, and $64 \% \mathrm{D}_{2} \mathrm{Se}$. Total hydrogen incorporation in this mixture is $23 \%$, which is far from the isotopic distribution of the solvent ( $3 \% \mathrm{H}$ and $97 \% \mathrm{D})$. The present findings contradict the previous hypothesis, which assumed that the isotopic composition of hydrogen selenide is similar to that of the solvent due to the rapid $\mathrm{H} / \mathrm{D}$ exchange (as a consequence of the strongly acid nature of $\mathrm{H}_{2} \mathrm{Se}$ and $\mathrm{D}_{2} \mathrm{Se}$ ) [1]. More experiments are needed to understand the mechanism controlling the isotopic composition of hydrogen selenide generated by borohydride from aqueous selenite solutions. Clearly, the deconvolution model for mass spectra will be of paramount importance for the realization of this difficult task.

\section{Conclusions}

We have shown that the concentration profiles of the overlapping isotopologues can be accurately extracted from their mixture mass spectra. Three conceptually different mathematical strategies are compared to achieve this aim (see Table 2). To our knowledge, this is the first attempt to investigate the quantitative aspects of the reconstruction of unknown isotopologue mass spectra. The above mentioned strategies can be employed to quantitatively assess isotopologue concentrations from their overlapping $70 \mathrm{eV}$ electron impact spectra and can be successfully extended to more complicated isotopologue systems, such as $\mathrm{AsH}_{3} / \mathrm{AsH}_{2} \mathrm{D}$ / $\mathrm{AsHD}_{2} / \mathrm{AsD}_{3}$, to study the hydride generation mechanism.

\section{Acknowledgments}

JM thanks the National Science and Engineering Research Council of Canada for the post-doctoral fellowship.

\section{References}

1. D'Ulivo, A.; Mester, Z.; Sturgeon, R. E. The Mechanism of Formation of Volatile Hydrides by Tetrahydroborate(III) Derivatization: A Mass Spectrometric Study Performed with Deuterium Labeled Reagents. Spectrochim. Acta B 2005, 60, 423-438.

2. Meija, J.; Caruso, J. A. Deconvolution of Isobaric Interferences in Mass Spectra. J. Am. Soc. Mass Spectrom. 2004, 15, 654-658.

3. Libnau, F. O.; Christy, A. A.; Kvalheim, O. M. Determination of the Equilibrium Constant and Resolution of the HOD Spectrum by Alternating Least-Squares and Infrared Analysis. Appl. Spectrosc. 1995, 49, 1431-1437.

4. Christy, A. A.; Egeberg, P. K. Quantitative Determination of Surface Silanol Groups in Silica Gel by Deuterium Exchange Combined with Infrared Spectroscopy and Chemometrics. Analyst 2005, 130, 738-744.

5. Trepat-Pere, E.; Lacorte, S.; Tauler, R. Solving Liquid Chromatography Mass Spectrometry Coelution Problems in the Analysis of Environmental Samples by Multivariate Curve Resolution. J. Chromatogr. A 2005, 1096, 111-122.

6. Zhang, H.; Garland, M.; Zeng, Y.; Wu, P. Weighted Two-Band Target Entropy Minimization for the Reconstruction of Pure Component Mass Spectra: Simulation Studies and the Application to Real Systems. J. Am. Soc. Mass Spectrom. 2003, 14, 1295-1305.

7. Shannon, C. E.; Weaver, W. The Mathematical Theory of Communication; University of Illinois Press: Chicago, 1949.

8. Guo, L.; Wiesmath, A.; Sprenger, P.; Garland, M. Development of 2D Band-Target Entropy Minimization and Application to the Deconvolution of Multicomponent 2D Nuclear Magnetic Resonance Spectra. Anal. Chem. 2005, 77, 1655-1662. 
1036

MEIJI ET AL.

J Am Soc Mass Spectrom 2006, 17, 1028-1036

9. Chew, W.; Widjaja, E.; Garland, M. Band-Target Entropy Minimization (BTEM): An Advanced Method for Recovering Unknown Pure Componeut Spectra. Application to the FTIR Spectra of Unstable Organometallic Mixtures. Organometallics 2002, 21, 1982-1990.

10. Widjaja, E.; Garland, M. Pure component spectral reconstruction from mixture data using SVD, global entropy minimization, and simulated annealing. Numerical investigations of admissible objective functions using a synthetic 7-species data set. J. Compute. Chem. 2002, 23, 911-919.

11. Widjaja, E.; Li, C.; Garland, M. Semi-Batch Homogeneous Catalytic In-Situ Spectroscopic Data. FTIR Spectral Reconstructions Using BandTarget Entropy Minimization (BTEM) Without Spectral Preconditioning. Organometallic 2002, 21, 1991-1997.

12. Widjaja, E.; Li, C.; Chew, W.; Garland, M. Band-Target Entropy Minimization. A Robust Algorithm for Pure Component Spectral Recovery. Application to Complex Randomized Mixtures of Six Components. Anal. Chem. 2003, 75, 4499-4507.

13. Press, W. H.; Teukolsky, S. A.; Vetterling, W. T.; Flannery, B. P. Numerical Recipes in C: The Art of Scientific Computing, and ed.; Cambridge University Press: Cambridge, 1992, pp 59-70.

14. Iwata, T.; Koshoubu, J. Minimization of Noise in Spectral Data. Apple. Spectrosc. 1996, 50, 747-752.

15. Iwata, T.; Koshoubu, J. New Method to Eliminate the Background Noise from a Line Spectrum. Apple. Spectrosc. 1994, 48, 1453-1456.

16. Corana, A.; Marchese, M.; Martini, C.; Ridella, S. Minimizing Multimodat Functions of Continuous Variables with the "Simulated Annealing" Algorithm. ACM Trans. Math. Soft. 1987, 13, 262-280.

17. Kirkpatrick, S.; Gelatt, D. D. J.; Vecchi, M. P. Optimization by simulated annealing. Science 1983, 220, 671-680.

18. Chang, Z. Prediction of Low-Energy Collision-Induced Dissociation Spectra of Peptides. Anal. Chem. 2004, 76, 3908-3922.

19. Thode, H. G. Isotopes. Ann. Rev. Phys. Chem. 1953, 4, 95-118.

20. Lawson, C. L.; Hanson, R. J. Solving Least Squares Problems; Prentice Hall: Englewood Cliffs, NJ, 1974.
21. Alfassi, Z. B. On the normalization of a mass spectrum for comparison of two spectra. J. Am. Soc. Mass Spectrom. 2004, 15, 385-387.

22. Wan, K. X.; Vidavsky, I.; Gross, M. L. Comparing similar spectra: From similarity index to spectral contrast angle. J. Am. Soc. Mass Spectrom. 2002, 13, 85-88.

23. Wolfsberg, M.; Mesa, A. A.; Pyper, J. W. Effect of Vibrational Anharmonicity on the Isotopic Self-Exchange Equilibria $\mathrm{H}_{2} \mathrm{X}+\mathrm{D}_{2} \mathrm{X}=2 \mathrm{HDX}$. J. Chem. Phys. 1970, 53, 3138-3146.

24. Polyakov, V. B.; Horta, J.; Cole, D. R. Isotopic Self-Exchange Reactions of Water: Evaluation of the Rule of the Geometric Mean in Liquid-Vapor Isotope Partitioning. J. Phys. Chem. A 2005, 109, 86428645.

25. Kresge, A. J.; Chang, Y. Hydrogen Isotope Disproportionation in Water and its Effect on Isotope-Exchange Equilibrium Constants Measured in Aqueous Solution. J. Chem. Phys. 1968, 49, 1439-1440.

26. Friedman, L.; Shiner, V. J. J. Experimental Determination of the Disproportionation of Hydrogen Isotopes in Water. J. Chem. Phys. 1966, 44, 4639-4640.

27. Duplan, J. C.; Mani, L.; Brunet, J. L. NMR determination of the equilibrium constant for the liquid $\mathrm{H}_{2} \mathrm{O}-\mathrm{D}_{2} \mathrm{O}$ mixture. Chem. Phys. Lett. 2005, 413, 400-403.

28. Fenby, D. V.; Chard, A. Enthalpy of the isotopic exchange reaction $\mathrm{H}_{2} \mathrm{O}$ $+\mathrm{D}_{2} \mathrm{O}=2 \mathrm{HDO}$. Aust. J. Chem. 1978, 31, 241-245.

29. Pyper, J. W.; Newbury, R. S.; Barton, G. W. J. Study of the Isotopic Disproportionation Reaction Between Light and Heavy Water Using a Pulsed-Molecular-Beam Mass Spectrometer. J. Chem. Phys. 1967, 46, 2253-2257.

30. Pyper, J. W.; Newburg, R. S. Hydrogen-Deuterium Self-Exchange in Hydrogen Sulfide and Hydrogen Selenide as Studied with a PulsedMolecular-Beam Quadrupole Mass Filter. J. Chem. Phys. 1970, 52, 1966-1971. 